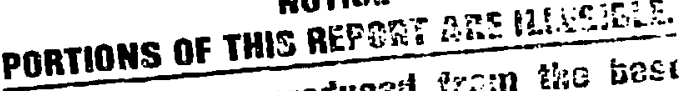
It has been reproduest iren the test avallable copy to permis sto brobiest possibie avellability.
LA-10264-MS

UC-34b

Issued: October 1984

LA- $-10264-H S$

DE85 005192

\title{
CYLTRAN Calculations of Electron Effects on XD Detectors
}

Stuart Mayer*

George J. Berzins

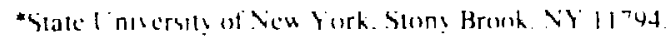

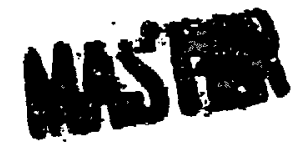

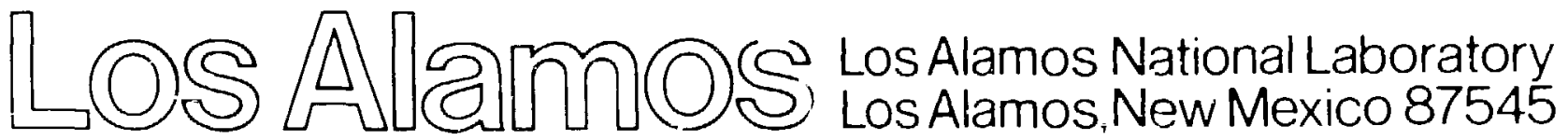




\section{DISCLAIMER}

This report was prepared as an account of woik sponsored by an agency of the United States Government. Neither the United States Government nor any agency thereof. nor any of their employes, inakes any warranty, express or implied, or assume any legal liability or responsibility for the accuracy, completeness, or usefulness of any information. apparatus, product, or process disclosed, or represents that its use would not infringe privately owned rights. Reference herein to any specific commercial product, process, or service by trade name, trademark, manufacturer, or otherwise does not necessarily constitute or imply its endorsement. recommendation, or favoring by the United States Government or any agency thercol. The views and opinions of authors expressed herein do not neressarily state or reflect those of the United States Government or any agency thereof. 
CYLTRAN CALCULATIONS OF ELECTRON EFFECTS ON XD DETECTORS

by

Stuart Mayer and George J. Berzins

ABSTRACT

Coupled electron-photon Monte Carlo calculations have been performed with the CYLTRAN code on a =ypical channel in a detector that has been flown aboard several spacecraft. The response of this channel has been examined as a function of incident electron energy over a range representative of background electrons in orbit. The signal is shown to arise from three separate reglons whose relative Importance changes with electron energy. Approximations used to cast the real problem into a format compatible with CYLTRAN are described.

\section{INTRODUCTION}

Background radiation effects tend to interfere to some degree with the operation of almost any type of instruments flown aboard eartr-orbiting satellites. Such radiation has been recognized for a long time and has been identified as consisting prirarily of electrons trapped in the magnetosphere. ${ }^{1}$ It is popularly referied to as Van Allen radiation in honor of its discoverer.

In some instances shielding can be implemented to protect instruments from these electrons. In other cases active discrimination techniques can be employed to reject unwanted signals. However, the undesired effects can rarely be completely suppressed and tend to become most annoying during magnetospheric disturbances when local electron fluxes can increase to 10-100 times their normal values. Unexpected, occasionally very large signals are sometimes recorded by a variety of detector systems. A large fraction of these observations appears to correlate with increases in background electron levels.

A variety of explanations have been advanced for these background signals. Among the more plausible are (1) cosmic ray showers following the striking of nearby, massive parts of the spacecraft by energetic primaries, (2) noise resulting from electrical breakdown following charging of the sparecraft by electron plasma, and (3) electron pileup in the detector. All may have some validity. The latter two would also tend to correlate with magnetic substorm activity. 
To contribute toward a better understandins, of electron interactions, we undertook a calculational study of one particular detector type, the XD, whose geometry is reasonably sultable for an existing code. That code, CYLTRAN, requires cylindrical symmetry and treats coupled electron-photon interactions. It was still necessary, however, to make several simplifyinr ipproximations.

In this study we have explored the effect of incident electron energy on the detector signal and have identifled and tabulated interactions in three different reglons of the detector that can contribute to the signal. Two of these regions are not significant when the detector is performing its design function. Thus on-ground calibrations are not directly applicable. We have also examined the dependence of the signal on the approximations that are necessary to fit the detector into the constraints of the CYLTRAN code.

\section{THE CYLTRAN CODE}

The CYLTRAN code ${ }^{2}$ is a specialized and simplified version of a more general set of codes written at Sandia National Laboratories in Albuquerque. These codes are described by their authors in several documents cited in Ref. 2 .

Briefly, CYLTRAN derives its name from transport of electrons and photons in cylindrical coordinates. The code treats particle energies ranging from a few keV to several MeV, though, as its authors note, it is least reliable when energles compare with $K$ edges of the materials involved. All particles and their resulting secondarles are followed until they elther escape through the problem's geometric boundarles, or lose sufficlent energy to drop below a present threshold (1.e. are stopped). For example, an incident beam of 10-MeV electrons may produce bremsstrahlung, energetic secondary electrons (delta rays), and Ionization in the target material. Each of these quanta, Including fluorescence $x$ rays, is statistically tracked until it (or its secondary particles) escapes or decreases in energy to the threshold level, say $20 \mathrm{keV}$.

Two of CYLTRAN's limitations are very important for the XD problems. First, the geometry through which the particles are transported not only must have cylindrical symmetry, but must also be completely describable by cylinders and planes. Shapes such as cones, for example, though cylindrically symmetric are not permitted and must be approximated by a tapered set of cylinders. However, the problem can be divided into many $(<100)$ zones consisting of different materials and subzories of the same material. The second 11mitation arises from the fact that the code accommodates only flve materials plus 
vacuum. Each materiai can consist of up to 10 different elements, and may reappear any number of times within the problem cylinder.

The code tabulates net energy and net charge that has been deposited in each zone. It also tallies the spectrum and angular distribution of escaping electrons and photons. The angular and spectral resolutions of the escaping particles are selected by the user. Output data are generally normalized to unit incident particle.

Other features of the code include an input spectrum (vs monoenergetic) option, as well as a pulse height analysis of the deposited energy. It is also possible to assign an incident direction (or random distribution of directions), and to define the beam diameter ranging from an infinitely thin pencil to full size of the total problem cylinder. For a more comprehensive discussion, the reader is referred to Ref. 2 .

\section{APPROXIMATION OF THE XD PROBLEM}

The XD detector consists of 16 different channels, one of which is shown 1) Fig. 1. That channel is representative of nearly half of the total, differing from the others only in window and scintillator details. Thus, we felt that from an examination of one or two channels it would be possible to draw some generalized inferences and to Identify the 1 imits within which our approach is feasible.

The design intent of the channel in Fig. l is for low-energy particles to enter through the window and to strike the scintillator. The resulting light from the scintillator is transmitted through the lead glass light pipe to the silicon photodiode where the optical energy is converted into an electronic signal for further processing and recording. The electronic signal is related to the energy deposited in the scintillator and thus to the energy (or flux) of the Incident particles.

ecause the CYLTRAN code does not accommodate cones, a useful approximation is to replace the conical collimator in Fig. I with a set of finely stepped cylinders where diameters follow the tapes of the cone. The approximation should be valid for incident electrons whose range in the material exceeds the step dimensions. Thus, the lower the electron energy, the finer is the step size required. For 0.1 - and $0.3-\mathrm{MeV}$ electrons, for example, steps in aluminum should be smaller than $0.07 \mathrm{~mm}$ and $0.4 \mathrm{~mm}$, respectively. This places a practical limit on the problem since CYLTRAN is restricted to $\leqslant 100$ zones. 


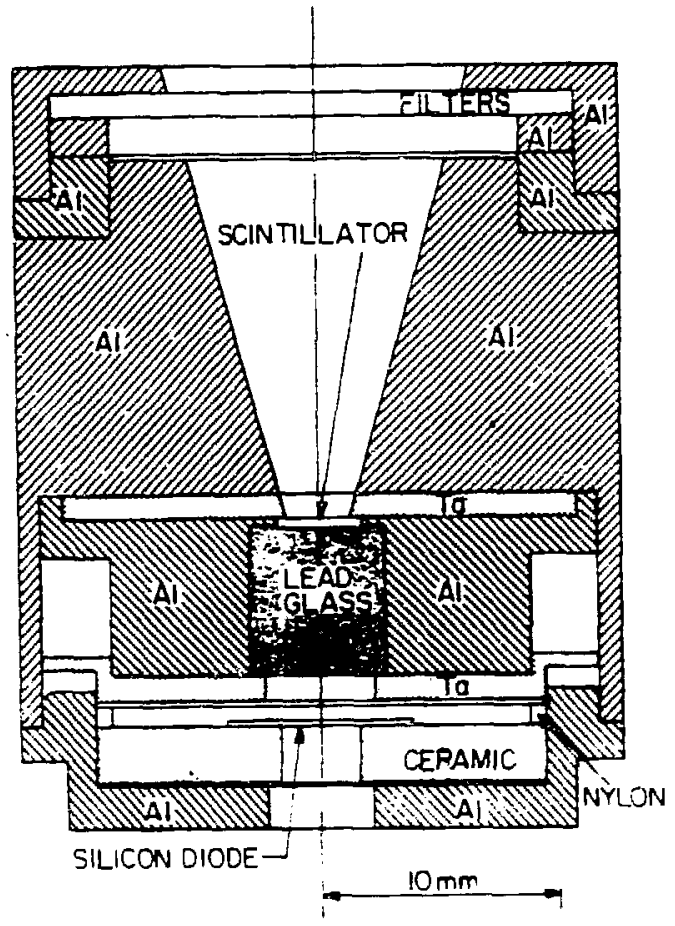

Fig. 1.

Outline drawing of a typical XD detector chaniel geometry.

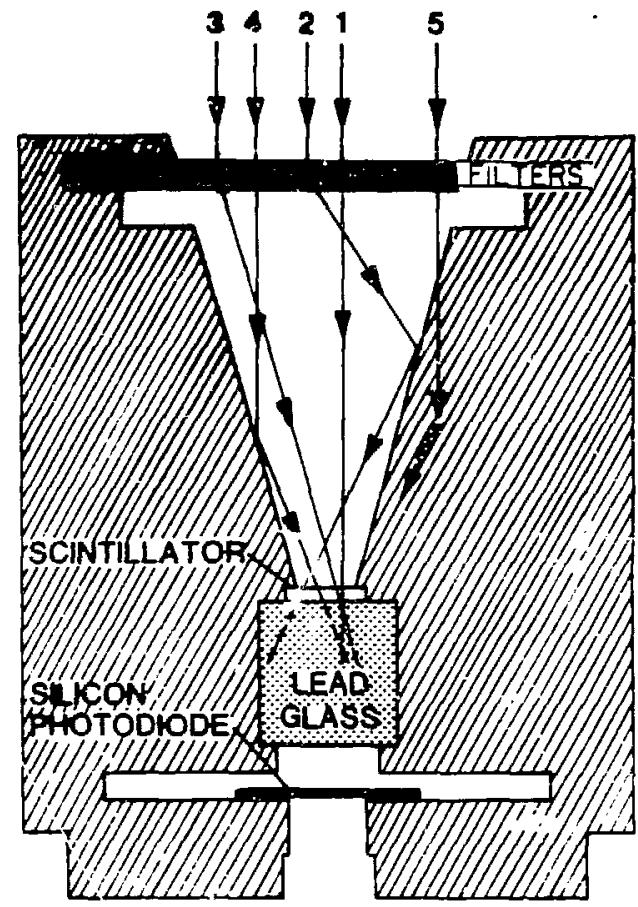

Fig. 2 .

Some of the paths by which energy from incident electrons can reach the scintillator (and the lead glass light pipe and silicon phot-iode) in an XDchannel.

We explored possible simplifications of the problem by considering various paths, shown as rays in Fig. 2, by which energy can raach the scintillator. Briefly, we compared the results among the following three cases: (1) an "oper:" geometry when the electrons were incicent within a cylinder whose diameter was that of the scintillator, and whose walls were infinitesimaliy thin: (2) a "confined" geometry that was identical to the first, except that the cylinder wall was of finite thickness; and (3) an "extended" geometry where the cy"tnder wall was thick and short, and was located to simulate various posittons 11 ong the cone. These geometries are illustrated in Fig. 3,

A brief study showed the results to be rather sensitive to details to these simplified geometries, especially at the higher electron energies. We therefore proceeded with the more complicated representation that involved the tapered set of cylinders and also included details of the lower part of the housing. The remafning two major departures from reality were (1) use of an equivalent circular rather than square outer cross section for the channel and (2) use of aluminum rather than silicon for the detector material. The 1atter arose from the five-material limit imposed by CYLTRAN. 


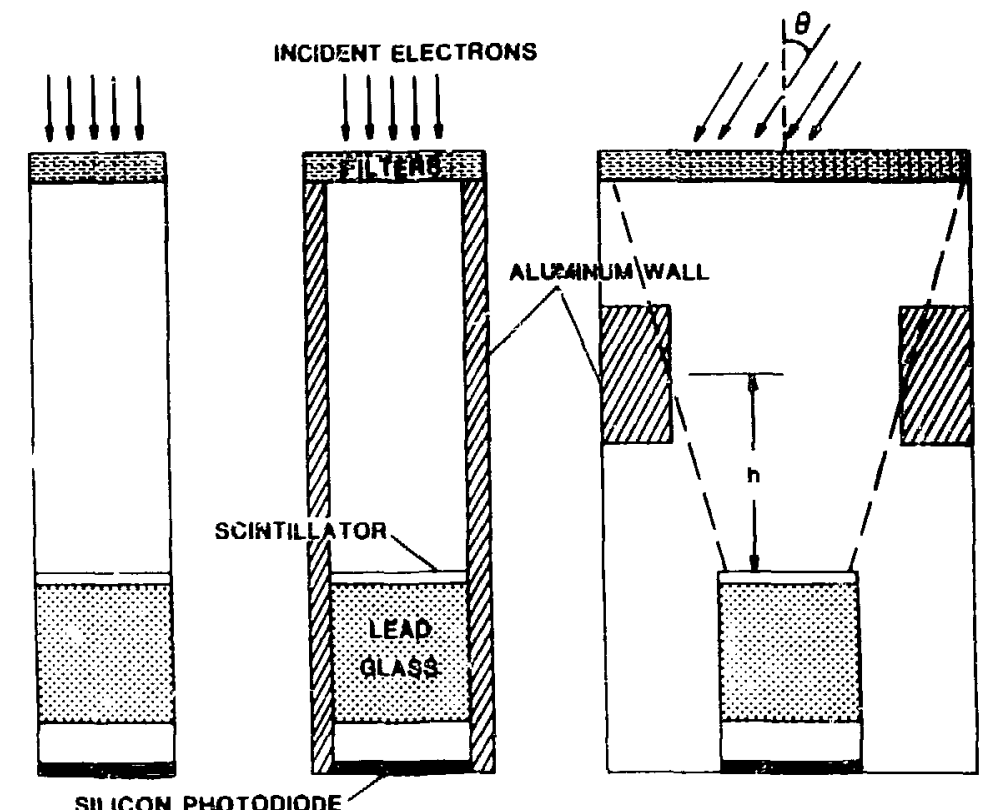
(a) OPEN
(b) CONFINED
(c) EXTENDED

Fig. 3.

Three simplified geometries considered as approximations to the geometries in Fig. 1 and used to explore the effects of the various paths shown in Fig. 2.

We limited the surface exposed to incident electrons to $1.1 \mathrm{~cm}^{2}$, just large enough to encompass the collimator opening, for practical reasons. Our Initial study of the simplified geometries in Fig. 3 demonstrated that most of the effect from scattering (i.e. rays 2, 3, and 4 of Fig. 2) was produced by a thin layer less than $1 \mathrm{~mm}$ from the surface. Contribution from thicker walls increased with electron energy (especlally above $10 \mathrm{MeV}$ ), as would be intuitively expected.

In the stepped geometry, CYLTRAN required several minutes of running time per Inctdent electron energy. To increase the problem cylinder thickness enough to match the actual detector would have dramatically increased the runring time, at little or no gain in accuracy below $\sim 10 \mathrm{MeV}$. At higher energies, however, a significant error may have been introduced by imposing this thickness 1 imit.

\section{RESULTS AND DISCUSSION}

Our primary interest is in the energy deposition in the scintillator, in the lead glass, and in the silicon photodiode. The recorded signal can result from a combination of light trom the scintillator, light from the lead glass, and direct energ; deposition in the silicon. In the first two regions, factors 
such as absorbed-to-optical energy-conversion efficiencies and geometric efficiencies must be consiciered. In the third region, the absorbed energy is directly converted to charge through well known processes.

The energy deposited in each of these thee regions is compared as a function of incident electron energy in Fig. 4. The stepped cylinder geometry was used for these calculations. The error bars in Fig. 4 represent worst case slatistical uncertainty. For all of the other points, the statistical error estimated by CYLTRAN was typically less than $5 \%$.

The significance of the detector housing is evident upon comparison of Fig. 4 with Fig. 5. The latter results from the "open" geometry calculation (see Fig. 3), aligned with only the full area of the scintillator. Note that even though the ordinate units in the two figures are the same, a direct comparison is misleading because the area encumpassed by the beam for Fig. 4 was 10.6 times greater than that for Fig. 5.

A usefi:l comparison can be obtalried as follows. Because the beam for Fig. 4 encompasses that for Fig. 5, but is 10 times larger, the part of the energy deposition owing to the direct incident electrons is that shown in Fig. 5, but scaled down by the area ratio. The remainder of the energy is deposited as a result of electrons that strike the surrounding material. These curves are overlaid in Fig. 6, the direct incidence data scaled by 10 .

The most striking resule of this comparison is that the surrounding materials account for more than half of the energy deposited in the scintillator and in the light pipe. In the case of the photodiode, about $90 \%$ is due to the surroundings. Several features of Figs. 4 and 5 agree with semiquantitarive, intuitive expectations. First, the energy deposition in the (thin) scintillator in Fig. 5 follows well-known dE/dx data. Second, energy deposition in the silicon increases rapidly above about 5 to $8 \mathrm{MeV}$, the incident electron energy whose range exceeds the thickness of the lead glass light plpe. The monotonic increase with energy occurs because of secondary interactions. The higher energy primary electrots generate showers that contain more secondary electrons as well as bremsstrahlung photons. Thus $1 \mathrm{t}$ is the shower that is incident on the photodiode, rather than essentially single, monoenergetic primailes as in the case of the scintillator.

Most of the energy (of that shown in Figs. 4 and 5) 1s deposited ir: the 1 ight pipe--a consequence of the light plpe's large volume and mass. Note that deposition in the 1 ight pipe increases slowly above $10 \mathrm{MeV}$, a result of the Increasing physical extent of the secordary shower and the attendant laterai escape. Note also the energy accounted for in Fig. 5 is less than the incident 


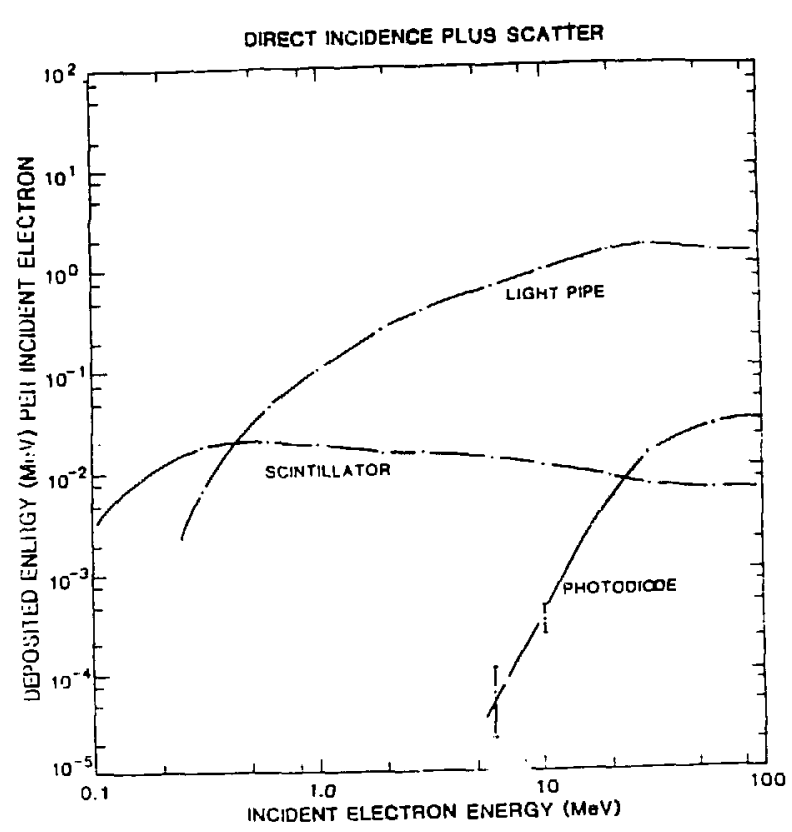

Fig. 4.

CYLTRAN calculations of energy deposited in the scintillator, tile light pipe, and the photodiode. The best approximation, the stepped geometry, was used.

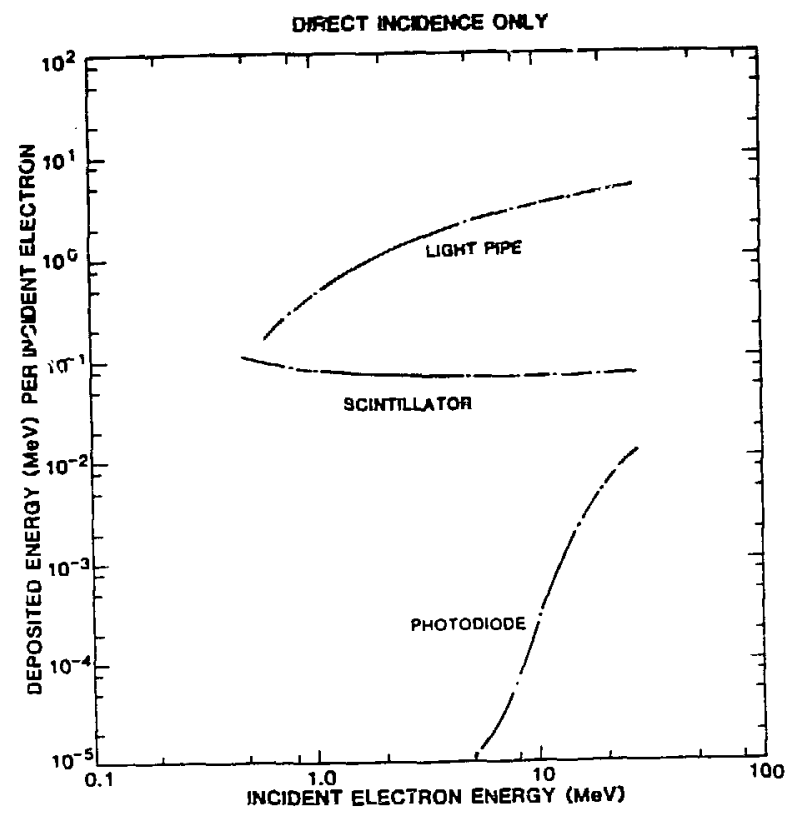

Fig. 5.

Same as Fig. 4, except that the great1y simplified, "open" geometry (see Fig. 3) was used.

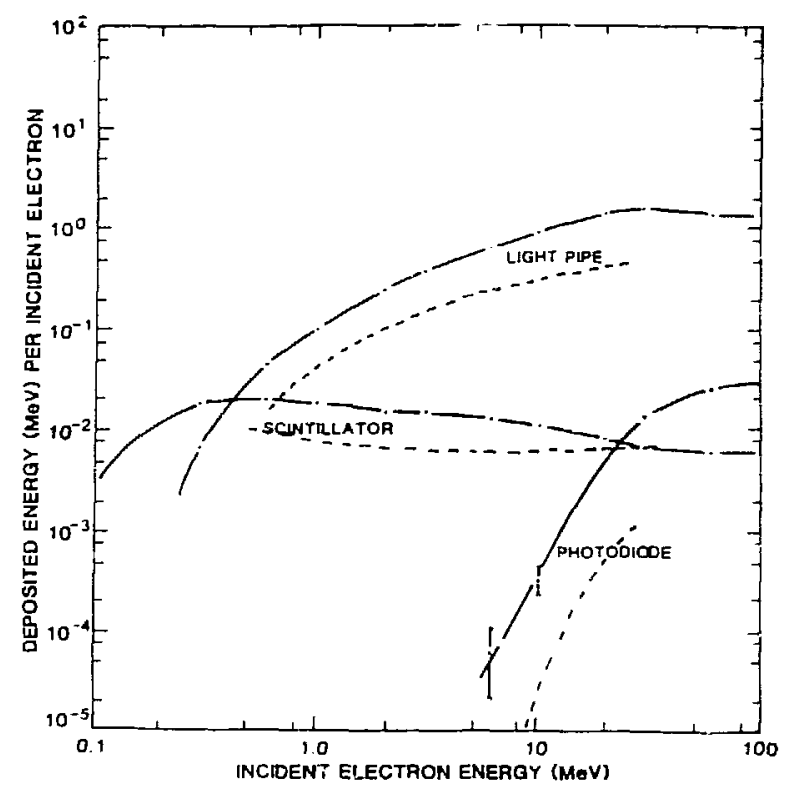

Fig. $\overline{\text {. }}$

Comparison between the results for the "stepped" and the "open" geometries. The data have beca scaled according to area of the incident beam. 
electron energy, again a result of secondary showers that permit some of the energy to escipe through the problem boundaries.

The total, observable signal Erom the detector can be expressed for each energy as

$$
Q(E)=k_{1} \Delta E_{S i}+k_{2}\left[\varepsilon_{S c} \Omega_{S C} \Delta E_{S C}+\varepsilon_{\ell} \Omega_{\ell} \Delta E_{\ell}\right]
$$

where the $\Delta E_{i}$ are the energy depositions in Fig. 4; $k_{1}$ and $k_{2}$ are the conversion efficiencies of absorbed energy to charge for incident ionizing radiation $\left(1.6 \times 10^{-19}\right.$ coul/3.6 eV $)$ and optical radiation $(0.3$ coul/joule $)$, respectively; $\varepsilon_{s c}$ and $\varepsilon_{\ell}$ are the absorbed energy-to-1ight conversion efficiencies $\left(\sim 0.05\right.$ and $10^{-2}-10^{-3}$ for the scintillator and 1 ig,ht pipe, respectively); and $\Omega_{\mathrm{sc}}$ and $\Omega_{\ell}$ are 1 ight collection efficiencies, estimated as $\sim 9 \%$ and $\sim 12 \%$ for the photodiode viewing the scintillator and the 1 ight pipe, r espectively.

It is instructive to evaluate the above expression for a range of incident electron energies, even without knowing the conversion efficiencles, etc., accurately. These results are summarized in Table I for the stepped geometry case (Fig. 4). We chose 0.003 for the light pipe scintillation efficiency.

The data in Table I show the scintillator contribution to be the most significant at low energies (below $1 \mathrm{MeV}$ ) and for direct interactions with the photodiode to dominate at very high $(>20 \mathrm{MeV})$ electron energles. The first of these conclusions is also suggested in Fig. 4, but the latter is not obvious in that figure. In the midrange of energies (1-20 MeV), all three regions contribute to the signal, the particular mix being very dependent on the values used for conversion and collection efficiencies.

7. CONCLUS IONS

We have used the one-dimensional Monte Carlo code CYLTRAN to calculate the energy deposited throughout the typical XD detector channel. The results show three regions--the scintillator, the light pipe, and the photodiode--that absorb energy and contribute to the signal from that channel. The relative importance of each region depends very much on the incident electron energy and on the actual values of energy-to-1ig!ı conversion efficiencies and on optical coupling. 
ESTIMATF.D SIGNAL PER INCIDENT ELECTRON

\begin{tabular}{|c|c|c|c|c|c|c|c|}
\hline \multirow{2}{*}{$\begin{array}{l}\text { Electron } \\
\text { Energy } \\
\text { (MeV) } \\
\end{array}$} & \multicolumn{3}{|c|}{ Deposited Energy $(\mathrm{MeV})$} & \multicolumn{4}{|c|}{ Predicted Signal (Coulomb $\times 10^{-17}$ ) } \\
\hline & Scint. & Light Pipe & Silicon & Scint. & Light Pipe & Silicon & Total \\
\hline 0.1 & 0.0043 & -- & -- & 0.93 & - & - & 0.93 \\
\hline 0.2 & 0.015 & 0.00033 & - & 3.2 & 0.00057 & - & 3.2 \\
\hline 9.5 & 0.022 & 0.035 & - & 4.8 & 0.60 & -- & 5.4 \\
\hline 1 & 0.017 & 0.10 & $-\infty$ & 3.7 & 1.7 & -- & 5.4 \\
\hline 2 & 0.016 & 0.25 & - & 3.5 & $\therefore$ & $\cdots$ & 7,8 \\
\hline 5 & 0.014 & 0.59 & 0.00003 & 3.0 & 10 & 0.13 & $13 . i$ \\
\hline 10 & 0.010 & 0.95 & 0.00076 & 2.2 & 16 & 3.4 & 21.6 \\
\hline 20 & 0.0077 & 1.50 & 0.0064 & 1.7 & 26. & 28. & 55.7 \\
\hline 50 & 0.0062 & 1.47 & 0.018 & 1.3 & 25. & 120 & 146. \\
\hline 100 & 0.0061 & 1.42 & $0.03 i_{i}$ & 1.3 & 24. & 150 & 175. \\
\hline
\end{tabular}

During the course of these calculations, we found that results were quite sensitive to simplifying assumptions. Thus we constructed the problem to include a large number of small zones to better apprisimate the actual geometry. This same geometry can be used for other channels in the detector, with appropriate changes for window and scintillator material details. We have performed some cursory calculations on a few of the other channels for a few electron energies. The results, though sketchy at this point, suggest that this technique holds promise for interpreting some of the backsround signals that have been observed in orbit.

\section{ACKNOWLE DGMENTS}

We are gratetul to M. M. Fikani and to J. M. Mack for helpful discussions and suggestions regarding the CYJ.TRAN code and to $W$. R. Scarlett and $J$ : R. Ashridge for their support of this project. S. Yaver thanks the Los Alamos National Laboratory and the Astronouy/Astrophysics (iroup in particular for making his participation in this projert possible through the Lahoratory's summer student program. 
REFERENCES

1. R. C. Haymes, Introdurtion to Space Science (John Wiley \& Sons, Inc., Now York, 1971).

2. J. A. Halbleib, Sr. and W. H. Vandevender, "CYLTRAN: A CylindricalGeometry Multimaterial Electron/Photon Monte Carlo Transport Code," Sandia National Laboratories report SAND 74-0030 (March 1975). 